MODELING, IDENTIFICATION AND CONTROL, 1996, VOL. 17, NO. 1, 17-26

doi:10.4173/mic.1996.1.2

\title{
Motion control of underwater vehicle-manipulator systems using feedback linearization
}

\author{
INGRID SCHJØLBERG† and OLAV EGELAND†
}

Keywords. Underwater robotics, feedback linearization

\begin{abstract}
In this paper control of underwater vehicle-manipulator systems using feedback linearization has been studied. Performance, robustness and energy consumption of the system depend on the choice of output variables, these output variables can be chosen in several ways. In this paper two alternatives have been analysed by simulations, decoupling of the manipulator end-effector velocities from the-vehicle velocities and from the -total system momentum. The performance is almost the same for the two choices of decoupling schemes while robustness and energy consumption of the system depend on the accuracy of the dynamic model.
\end{abstract}

\section{Introduction}

Semi-autonomous vehicle-manipulator systems are useful for space and underwater operations. To be able to perform operations like inspection, maintenance, repair and service work on space and underwater installations, accurate control of both the vehicle and the manipulator end-effector is essential.

The feedback linearization technique (Spong and Vidyasagar, 1989) is a common approach to control design of robotic systems. The concept is to choose an appropriate feedback loop so that the nonlinearities in the system are cancelled. This results in a linear system for which many control techniques are available. The disadvantage with the feedback linearization method is that exact knowledge of the system dynamics is required. Nevertheless, the simplicity of the technique and the possibility of using linear control techniques makes the feedback linearization method attractive.

Feedback linearization has been utilized to control spacecraft-manipulator systems, see for instance Dubowsky and Papadopoulos (1993) who decoupled the spacecraft position and attitude vector from the manipulator position and orientation vector. Egeland and Sagli (1993) suggested decoupling the manipulator motion and the system total linear and angular momentum. This resulted in a more energy-efficient control scheme.

Controllers based on the feedback linearization technique may also be applied to underwater vehicle-manipulator systems. However, these systems are affected by hydrodynamical forces and possess uncertainties in the hydrodynamical coefficients and damping terms. It is therefore necessary to study the performance and robustness of the control schemes based on feedback linearization. In this paper the decoupling schemes suggested in Egeland and Sagli (1993) are applied to an underwater vehicle-manipulator system and the schemes are evaluated by simulations, considering robustness, performance, energy consumption and implementation simplicity.

Received 20 August 1995

$\dagger$ University of Trondheim, The Norwegian Institute of Technology, Department of Engineering Cybernetics, Norway.

Presented at the 3rd IFAC Workshop on Control Applications in Marine Systems, Trondheim, Norway, May 1995 (CAMS ’95). 
This paper is organized as follows: in Section 2 the dynamics and kinematics of the vehicle--manipulator are retrieved. The control laws are reformulated in Section 3, and a simulation study of the closed loop systems is presented in Section 4 with the conclusions in Section 5.

\section{System dynamics and kinematics}

The equations of motion for an underwater vehicle-manipulator system presented in Schjølberg and Fossen (1994) are summarized in the following section.

\subsection{Equations of motion for vehicle-manipulator systems}

The equations of motion of an underwater manipulator attached to an underwater vehicle can be written in the form

$$
\begin{aligned}
\boldsymbol{M}_{m}(\boldsymbol{q}) \ddot{\boldsymbol{q}}+\boldsymbol{C}_{m}(\boldsymbol{q}, \dot{\boldsymbol{q}}) \dot{\boldsymbol{q}}+\boldsymbol{D}_{m}(\boldsymbol{q}, \dot{\boldsymbol{q}}, \boldsymbol{v}) \dot{\boldsymbol{q}}+\boldsymbol{g}_{m}(\boldsymbol{q})+\boldsymbol{M}_{c}(\boldsymbol{q}) \dot{\boldsymbol{v}}+\boldsymbol{C}_{3}(\boldsymbol{q}, \dot{\boldsymbol{q}}, \boldsymbol{v}) \\
+\boldsymbol{D}_{3}(\boldsymbol{q}, \dot{\boldsymbol{q}}, \boldsymbol{v})=\tau_{m}
\end{aligned}
$$

where $\boldsymbol{q} \in \mathbb{R}^{n_{1}}$ is the vector of generalized coordinates for the manipulator and $\tau_{m} \in \mathbb{R}^{n_{1}}$ is the manipulator control force vector and $n_{1}$ is the number of manipulator joints. The vector $v$ is the vector of generalized vehicle velocities in a body-fixed frame. The matrix $\boldsymbol{M}_{m} \in \mathbb{R}^{n_{1} \times n_{1}}$ is the matrix of inertia and added inertia, $\boldsymbol{C}_{m} \in \mathbb{R}^{n_{1} \times n_{1}}$ is the matrix of Coriolis and centripedal terms, $\boldsymbol{D}_{m} \in \mathbb{R}^{n_{1} \times n_{1}}$ is the matrix of hydrodynamical damping terms and $\boldsymbol{g}_{m} \in \mathbb{R}^{n_{1}}$ is the vector of gravity and buoyant forces. The matrix $\boldsymbol{M}_{c}$ is due to the reaction force between the vehicle and the manipulator and the matrices $\boldsymbol{C}_{3}$ and $D_{3}$ are the Coriolis and centripetal terms and the damping effects on the manipulator due to the vehicle motion. Similarly the equations of motion for an underwater vehicle holding a manipulator can be written, in a vehicle-fixed reference frame

$$
M_{v} \dot{v}+C_{v}(v) v+D_{v}(v) v+g_{v}(\eta)+f(q, \dot{q}, \ddot{q}, v)=\tau_{v}
$$

where $\boldsymbol{v} \in \mathbb{R}^{n_{2}}$ and $\boldsymbol{\eta} \in \mathbb{R}^{m}$. According to the SNAME notation $v=[u, v, w, p, q, r]^{\mathrm{T}}$ is the vector of linear and angular velocities. The vector $\boldsymbol{\eta}$ is the position/attitude vector of the vehicle in the inertial reference frame (I-frame). The scalars $n_{2}$ and $m$ are the vehicle degrees of freedom in the body-fixed and earth-fixed reference frame. The vector $\tau_{v} \in \mathbb{R}^{n_{2}}$ is the vector of control forces and moments and the vector $f \in \mathbb{R}^{n_{2}}$ are the forces and moments applied by the manipulator on its base. The matrix $\boldsymbol{M}_{v} \in \mathbb{R}^{n_{2}}$ is the inertia and added inertia matrix, $C_{v}(v) \in \mathbb{R}^{n_{2} \times n_{2}}$ is the matrix of Coriolis and centripetal terms, $\boldsymbol{D}_{v}(\boldsymbol{v}) \in \mathbb{R}^{n_{2} \times n_{2}}$ is the hydrodynamic damping matrix and $\boldsymbol{g}_{v}(\boldsymbol{\eta}) \in \mathbb{R}^{n_{2}}$ is the vector of gravity and buoyant forces. The reaction forces and moments $f$ applied by the manipulator on its base can be written in the form

$$
\begin{gathered}
f(q, \dot{q}, \ddot{q}, v)=H(q) \dot{v}+C_{1}(q, \dot{q}, v) v+D_{1}(q, \dot{q}, v) v+M_{c}(q) \ddot{q}+C_{2}(q, \dot{q}) \dot{q} \\
+D_{2}(q, \dot{q}, v) \dot{q}+g_{E}
\end{gathered}
$$

The matrices $\boldsymbol{M}_{c}(\boldsymbol{q})=\boldsymbol{M}_{c}^{\mathrm{T}}$ are the terms due to the reaction forces between the vehicle and manipulator and $\boldsymbol{H}(\boldsymbol{q})=\boldsymbol{H}^{\mathbf{T}}$ is the added inertia due to the mass of the manipulator. The matrices $\boldsymbol{C}_{i}$ contain the Coriolis and centripetal terms and $\boldsymbol{D}_{i}$ contains the damping terms due to the interaction between the manipulator and the vehicle rigid body. The vector $g_{E}(q)$ is the increased gravitational forces and moments due to the manipulator motion. 


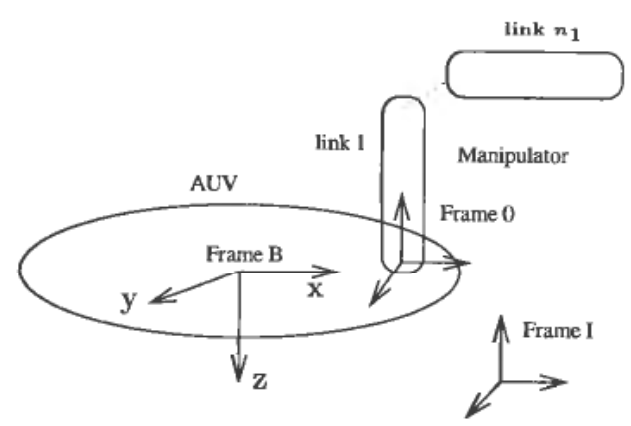

Figure 1. Vehicle-manipulator system.

Combining the equations of motion of the manipulator and the vehicle yields the total dynamic model in a vehicle-fixed frame, located at the manipulator base

$$
M(q) \dot{u}+C(q, u) u+D(q, u) u+g\left(q, \eta_{2}\right)=\tau
$$

where $u=\left[v^{T}, \dot{q}^{T}\right]^{T}$ is the vector of generalized velocities. The system matrices are

$$
\begin{aligned}
\boldsymbol{M}(\boldsymbol{q}) & =\left[\begin{array}{ll}
\boldsymbol{M}_{v}+\boldsymbol{H}(q) & \boldsymbol{M}_{c}(\boldsymbol{q}) \\
\boldsymbol{M}_{c}^{\mathrm{T}}(\boldsymbol{q}) & \boldsymbol{M}_{m}(\boldsymbol{q})
\end{array}\right] \\
\boldsymbol{C}(\boldsymbol{q}, \boldsymbol{u}) & =\left[\begin{array}{ll}
\boldsymbol{C}_{v}(\boldsymbol{v})+\boldsymbol{C}_{1}(\boldsymbol{q}, \dot{\boldsymbol{q}}, \boldsymbol{v}) & \boldsymbol{C}_{2}(\boldsymbol{q}, \dot{\boldsymbol{q}}) \\
\boldsymbol{C}_{3}(\boldsymbol{q}, \dot{\boldsymbol{q}}, \boldsymbol{v}) & \boldsymbol{C}_{m}(\boldsymbol{q}, \dot{\boldsymbol{q}})
\end{array}\right] \\
\boldsymbol{D}(\boldsymbol{q}, \boldsymbol{u}) & =\left[\begin{array}{ll}
\boldsymbol{D}_{v}(\boldsymbol{v})+\boldsymbol{D}_{1}(\boldsymbol{q}, \dot{\boldsymbol{q}}, \boldsymbol{v}) & \boldsymbol{D}_{2}(\boldsymbol{q}, \dot{\boldsymbol{q}}, \boldsymbol{v}) \\
\boldsymbol{D}_{3}(\boldsymbol{q}, \dot{\boldsymbol{q}}, \boldsymbol{v}) & \boldsymbol{D}_{4}(\boldsymbol{q}, \dot{\boldsymbol{q}}, \boldsymbol{v})
\end{array}\right] \\
\boldsymbol{g}(\boldsymbol{q}, \boldsymbol{\eta}) & =\left[\begin{array}{l}
\boldsymbol{g}_{v}(\boldsymbol{\eta})+\boldsymbol{g}_{E}(\boldsymbol{q}) \\
\boldsymbol{g}_{m}(\boldsymbol{q})
\end{array}\right] \\
\boldsymbol{\tau} & =\left[\boldsymbol{\tau}_{v}^{\mathrm{T}}, \tau_{m}^{\mathrm{T}}\right]^{\mathrm{T}}
\end{aligned}
$$

The total system has $n_{1}+n_{2}$ degrees of freedom.

\subsection{Kinematic equations}

There are several working tasks for underwater vehicle--manipulator systems. The tasks demand the control of the manipulator end-effector position and orientation vector $\boldsymbol{x}_{\boldsymbol{e}}$ and the vehicle position and attitude vector $\boldsymbol{\eta}$. The manipulator end-effector position and orientation in the base-frame 0 is denoted ${ }^{0} x_{e}$. The end-effector velocity vector can be written

$$
{ }^{0} \dot{\boldsymbol{x}}_{e}=\left[\begin{array}{c}
{ }^{0} \boldsymbol{v}_{e} \\
{ }^{0} \omega_{e}
\end{array}\right]=\boldsymbol{J}_{1} \boldsymbol{v}+\boldsymbol{J}_{2} \dot{\boldsymbol{q}}
$$

where

$$
\begin{aligned}
& \boldsymbol{J}_{1}=\left[\begin{array}{cc}
\boldsymbol{I}_{3 \times 3} & \boldsymbol{\alpha}_{1} \\
\mathbf{0} & \boldsymbol{I}_{3 \times 3}
\end{array}\right] \\
& \boldsymbol{J}_{2}=\left[\begin{array}{ccc}
\boldsymbol{\alpha}_{1} \boldsymbol{\beta}_{1} & \ldots & \boldsymbol{\alpha}_{n_{1}} \boldsymbol{\beta}_{n_{1}} \\
\boldsymbol{\beta}_{1} & \ldots & \boldsymbol{\beta}_{N}
\end{array}\right]
\end{aligned}
$$

and $\boldsymbol{\alpha}_{k}=-\sum_{j=k}^{n_{1}} S\left(\boldsymbol{R}_{0}^{j} d_{j l y-1}\right)$ and $\boldsymbol{\beta}_{k}=\boldsymbol{R}_{\mathrm{U}}^{k-1} z$. The matrix $\boldsymbol{S}$ is a skew-symmetric matrix 
operator defined such that: $\boldsymbol{a} \times \boldsymbol{b}=\boldsymbol{S}(\boldsymbol{a}) \boldsymbol{b}$. The vector $\boldsymbol{d}_{j / j-1}$ is the length of manipulator $\operatorname{arm} j$ and $z$ is the unit vector along the $\mathrm{z}$-axis.

The kinematic transformation between the I-fixed and vehicle-fixed reference frame is given by $\dot{\boldsymbol{\eta}}=\boldsymbol{J}_{v}\left(\boldsymbol{\eta}_{2}\right) \boldsymbol{v}$ (Fossen, 1994), where

$$
\boldsymbol{J}_{v}\left(\boldsymbol{\eta}_{2}\right)=\left[\begin{array}{cc}
\boldsymbol{J}_{v 1} & \mathbf{0} \\
\mathbf{0} & \boldsymbol{J}_{v 2}
\end{array}\right]
$$

and $\boldsymbol{\eta}=\left[\boldsymbol{\eta}_{1}^{\mathrm{T}}, \boldsymbol{\eta}_{2}^{\mathrm{T}}\right]^{\mathrm{T}}, \boldsymbol{\eta}_{1}$ is the position vector and $\boldsymbol{\eta}_{2}$ is the attitude vector. The end-effector velocity vector ${ }^{0} \dot{\boldsymbol{x}}_{e}$ can be expressed in the I-frame

$$
{ }^{I} \dot{\boldsymbol{x}}_{e}=\boldsymbol{R}_{\boldsymbol{I}}^{00} \dot{\boldsymbol{x}}_{e}
$$

where

$$
\boldsymbol{R}_{I}^{0}=\left[\begin{array}{cc}
\boldsymbol{J}_{v 1} & \mathbf{0} \\
\mathbf{0} & \boldsymbol{I}
\end{array}\right]
$$

and $\boldsymbol{I}$ is the $3 \times 3$ identity matrix.

\subsection{System momentum}

The system motion can be described in terms of the system momentum. The linear and angular momentum of a system of rigid-bodies are defined (Hughes 1986)

$$
\boldsymbol{p}=\sum_{i=0}^{n_{1}} m_{i} \boldsymbol{v}_{i} \quad \text { and } \quad \boldsymbol{h}=\sum_{i=0}^{n_{1}} \boldsymbol{I}_{i} \omega_{i}+\boldsymbol{r}_{i} \times \boldsymbol{p}_{i}
$$

where $m_{i}$ is the mass of body $i, v_{i}$ is the linear velocity of body $i, \boldsymbol{I}_{i}$ is the moment of inertia, $\omega_{i}$ is the angular acceleration $r_{i}$ is the position of the end-point of body $i$ and $\boldsymbol{p}_{i}$ is the linear momentum of body $i$. The external forces acting on the system are the control forces $\tau$, and the hydrodynamical forces, the Coriolis and centrifugal forces and the gravity and buoyant forces denoted $n(q, u, \dot{u})$. The system momentum can be written as a function of the system velocities (Sagli, 1991)

$$
\left[\begin{array}{c}
{ }^{0} \boldsymbol{p} \\
{ }^{0} \boldsymbol{h}
\end{array}\right]=\boldsymbol{P}_{01} \boldsymbol{v}+\boldsymbol{P}_{02} \dot{\boldsymbol{q}}
$$

The change in the system linear and angular momentum can be expressed

$$
\left[\begin{array}{l}
\dot{\boldsymbol{p}} \\
\dot{\boldsymbol{h}}
\end{array}\right]=\boldsymbol{\tau}-\boldsymbol{n}
$$

\section{Case study}

In the following section feedback linearization is applied to an underwater vehicle and manipulator system and two control laws are given for control of the vehicle and the manipulator end-effector.

\subsection{Control laws}

Feedback linearization of the vehicle dynamics is achieved by defining a controller in the form

$$
\boldsymbol{\tau}=\boldsymbol{M}(\boldsymbol{q}) \boldsymbol{a}_{\boldsymbol{u}}+\boldsymbol{C}(\boldsymbol{q}, \boldsymbol{u}) \boldsymbol{u}+\boldsymbol{D}(\boldsymbol{q}, \boldsymbol{u}) \boldsymbol{u}+\boldsymbol{g}\left(\boldsymbol{q}, \boldsymbol{\eta}_{2}\right)
$$

This concept assumes that the vehicle velocities and the joint velocities are measured 
as well as the vehicle position and attitude and the manipulator joint angles. Applied to the equations of motion (4) for the total system, yields the linearized system

$$
\dot{\boldsymbol{u}}=\boldsymbol{a}_{u}
$$

The augmented task velocity vector $\boldsymbol{y}$ is chosen according to the requirements of the task and can be written $\boldsymbol{y}=\boldsymbol{P}_{0} \boldsymbol{u}$ where $\boldsymbol{P}_{0}$ is a Jacobian defined according to the task. The time derivative $\dot{\boldsymbol{y}}$ is

$$
\dot{\boldsymbol{y}}=\boldsymbol{P}_{0} \dot{\boldsymbol{u}}+\dot{\boldsymbol{P}}_{0} \boldsymbol{u}
$$

This gives

$$
\begin{aligned}
\dot{\boldsymbol{u}} & =\boldsymbol{P}_{0}^{-1}\left(\dot{\boldsymbol{y}}-\dot{\boldsymbol{P}}_{0} \boldsymbol{u}\right)=\boldsymbol{a}_{u} \\
\dot{\boldsymbol{y}} & =\boldsymbol{P}_{0} \boldsymbol{a}_{u}+\dot{\boldsymbol{P}}_{0} \boldsymbol{u}=\boldsymbol{a}_{y}
\end{aligned}
$$

and the control vector

$$
\boldsymbol{a}_{u}=\boldsymbol{P}_{0}^{-1}\left(\boldsymbol{a}_{y}-\dot{\boldsymbol{P}}_{0} \boldsymbol{u}\right)
$$

This yields the closed loop error dynamics of the linearized system

$$
\dot{\boldsymbol{y}}-\boldsymbol{a}_{\boldsymbol{y}}=\mathbf{0}
$$

Two choices for the task velocity vector $\boldsymbol{y}$ are:

(1) Decoupling the manipulator end-effector velocities from the vehicle velocities by defining

$$
\boldsymbol{y}=\left[\begin{array}{c}
{ }^{\prime} \dot{\boldsymbol{x}}_{\boldsymbol{e}} \\
\dot{\boldsymbol{\eta}}
\end{array}\right]
$$

This is a scheme utilized in the control of spacecraft-manipulator systems. Tracking convergence of the system position/attitude and velocities is achieved using the control law

$$
\boldsymbol{a}_{y_{1}}=\left[\begin{array}{l}
\ddot{\boldsymbol{x}}_{e d}-\boldsymbol{K}_{p 1} \tilde{\boldsymbol{x}}_{e}-\boldsymbol{K}_{d 1} \dot{\tilde{\boldsymbol{x}}}_{e} \\
\ddot{\boldsymbol{\eta}}_{d}-\boldsymbol{K}_{p 2} \tilde{\boldsymbol{\eta}}-\boldsymbol{K}_{d 2} \dot{\tilde{\boldsymbol{\eta}}}
\end{array}\right]
$$

where $\tilde{\boldsymbol{\eta}}$ is the vehicle position/attitude error vector $\tilde{\boldsymbol{\eta}}=\boldsymbol{\eta}-\boldsymbol{\eta}_{d}$. The superscript $d$ indicates the desired value. The vector $\tilde{\boldsymbol{x}}_{e}$ is the manipulator position/orientation error vector $\tilde{\boldsymbol{x}}_{e}={ }^{\prime} \boldsymbol{x}_{e}-{ }^{\prime} \boldsymbol{x}_{e_{d} \text {. }}$ The end-effector velocity error vector is defined ${ }^{I} \tilde{\boldsymbol{x}}_{\boldsymbol{e}}=\left[{ }^{I} \tilde{\boldsymbol{v}}_{\boldsymbol{e}}^{\mathrm{T}}{ }^{0} \tilde{\boldsymbol{\omega}}_{\mathrm{e}}^{\mathrm{T}}\right]^{\mathrm{T}}$.

Remark 1. The manipulator controller is able to compensate for unpredicted vehicle motion due to the control of the end-effector in the I-frame. The vehicle thrusters are used to compensate for the reaction forces due to the manipulator motion.

(2) Egeland and Sagli (1993) suggested for a spacecraft-manipulator system to decouple the end-effector motion from the total system momentum to reduce energy consumption. Decoupling of the manipulator end-effector velocities from the total system momentum is achieved by defining the task velocity vector

$$
\boldsymbol{y}=\left[\begin{array}{c}
{ }^{I} \dot{\boldsymbol{x}}_{e} \\
{ }^{0} \boldsymbol{p} \\
{ }^{0} \boldsymbol{h}
\end{array}\right]
$$


The system motion can be controlled using the control law

$$
\boldsymbol{a}_{y 2}=\left[\begin{array}{c}
\ddot{\boldsymbol{x}}_{e d}-\boldsymbol{K}_{p 1} \tilde{\boldsymbol{x}}_{e}-\boldsymbol{K}_{d 1} \dot{\tilde{\boldsymbol{x}}}_{e} \\
\boldsymbol{P}_{01}\left(\ddot{\boldsymbol{\eta}}_{d}-\boldsymbol{K}_{p 2} \tilde{\boldsymbol{\eta}}-\boldsymbol{K}_{d 2} \dot{\tilde{\boldsymbol{\eta}}}\right)
\end{array}\right]
$$

Remark 2. From (9) it can be seen that the control vector has to counteract the hydrodynamical friction forces to be able to change the system momentum.

Integral action can be easily obtained by including the term $\int_{0}^{t} \tilde{z} \mathrm{~d} \tau$ (where $z$ is the position/attitude error vector) in the controllers. Scheme (18) is computationally more efficient than scheme (20) since the last scheme demand the computation of the Jacobians $\boldsymbol{P}_{01}$ and $\boldsymbol{P}_{02}$.

\section{Simulation study}

The objective of the simulation study was to evaluate the performance, energy consumption and robustness of the closed loop system utilizing the controllers (18) and (20).

A simulation model of an underwater vehicle carrying a planar two-arm manipulator system was implemented in Matlab. The model of the vehicle was based on the theoretical model of the Norwegian Experimental Remotely Operated Vehicle (NEROV) (Fossen, 1991), with vehicle weight set to $185 \mathrm{~kg}$. The manipulator links were assumed to be cylindrical with diameter $d=0.1 \mathrm{~m}$ and length $l=0.5 \mathrm{~m}$. The dry mass of a manipulator link was set to $25 \mathrm{~kg}$. The hydrodynamical damping was modeled as a sum of linear skin-friction, quadratic drag, lift force and rotational damping. The added inertia matrix was assumed to be diagonal. The hydrodynamical friction coefficients were functions of the angle of attack $\alpha$, angle of sideslip $\beta$ and the Reynold number (Schjølberg and Fossen, 1994). It was assumed that the fluid had Reynolds number $\mathrm{Rn}=10^{5}$, drag and lift coefficients $C_{D}=1 \cdot 1 \alpha, C_{L}=0 \cdot 25 \alpha$, linear skin friction coefficient $D_{s}=0 \cdot 1$ and rotational damping coefficients $C_{l p}=-0 \cdot 1 \alpha$, $C_{m q}=-0 \cdot 1 \alpha$ and $C_{n r}=-2 \beta$. The Strouhal number was chosen equal to 0.2 and the phase angle $\gamma=0$. The flow velocity was generated by a 1st order Gauss-Markov Process $\dot{V}_{f}(t)=w(t)-\frac{1}{T} V_{f}(t)$, weie $w(t)$ was a zero mean Gaussian white noise process and $T$ was the sampling time. Further, $V_{f}$ was limited by $0 \cdot 1 \leqslant V_{f} \leqslant 0 \cdot 6$.

The end-effector reference was chosen as a sinusoidal signal in the $\mathrm{x}$ - and $\mathrm{z}$-direction

$$
r=1+\sin (0 \cdot 5 \pi t)
$$

and with a constant component in the y-direction. The end-effector orientation was chosen to be constant. The redundancy resolution produced the vehicle reference in the $\mathrm{x}-, \mathrm{y}$ - and $\mathrm{z}$-direction. The vehicle attitude vector was chosen as

$$
\boldsymbol{\eta}_{2}=[0 \cdot 1,0 \cdot 1,0 \cdot 1]^{\mathrm{T}}
$$

\begin{tabular}{cccc}
\hline$K_{p_{11}}$ & $K_{p_{12}}$ & $K_{p_{21}}$ & $K_{p_{22}}$ \\
\hline $100 I$ & $100 I$ & $I$ & $I$ \\
$K_{d_{11}}$ & $K_{d_{12}}$ & $K_{d_{21}}$ & $K_{d_{22}}$ \\
$20 I$ & $20 I$ & $2 I$ & $2 I$
\end{tabular}

Table 1. Parameters in the control laws. 


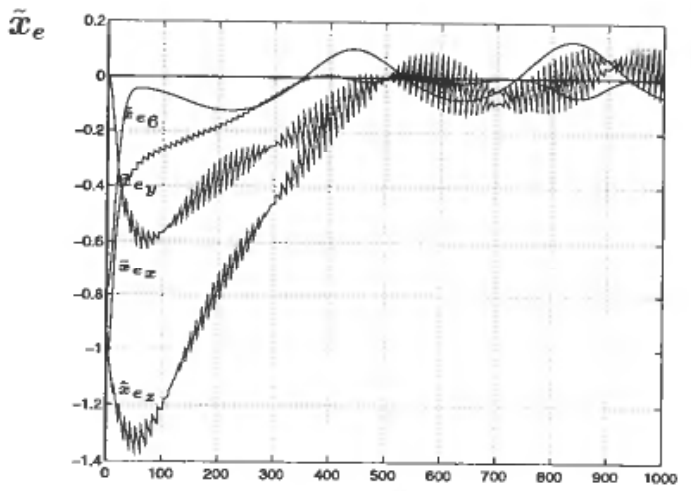

Figure 2. Simulation of the end-effector position/orientation error using (18).

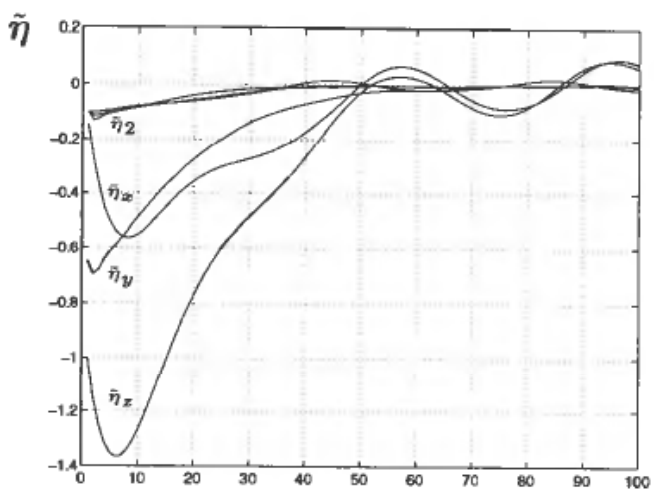

Figure 3. Simulation of the vehicle position/attitude error using (18).

All state variables were initially zero. The vehicle and manipulator dynamics were simulated using the 4 th order Runge-Kutta method with sampling time equal to $0 \cdot 1$ for the vehicle dynamics and sampling time equal to 0.01 for the manipulator dynamics.

The controller (18) was tuned under the requirement of high tracking accuracy and speed, and assuming exact knowledge of the system model, see Table 1 . The tracking error of the end-effector position/orientation vector and the vehicle position and attitude vector with controllers (18) and (20) are shown in Figs. 2-5.

The performance was evaluated by computing

$$
\begin{aligned}
& \boldsymbol{J}_{1}=\frac{1}{k} \sum_{i=1}^{k} \tilde{\boldsymbol{\eta}}_{i}^{\mathrm{T}} \tilde{\boldsymbol{\eta}}_{i} \\
& \boldsymbol{J}_{2}=\frac{1}{k} \sum_{i=1}^{k} \tilde{\boldsymbol{x}}_{\boldsymbol{e}_{i}^{\mathrm{T}}}^{\mathrm{T}} \tilde{\boldsymbol{x}}_{\boldsymbol{e}_{i}}
\end{aligned}
$$

where $k$ was the number of samples. The amount of vehicle control torque required in the $\mathrm{x}-, \mathrm{y}$ - and $\mathrm{z}$-directions was compared by calculating

$$
\begin{aligned}
& J_{3}=\sum_{i=1}^{k}\left|\tau_{1_{i}}\right| \\
& J_{4}=\sum_{i=1}^{k}\left|\tau_{z_{i}}\right|
\end{aligned}
$$




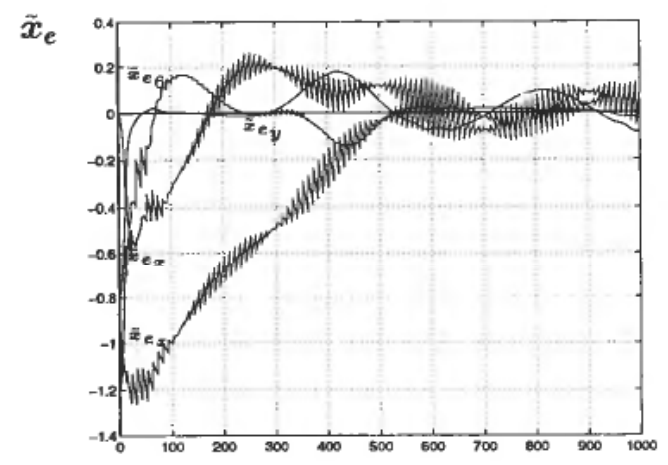

Figure 4. Simulation of the end-effector position/orientation error using (20).

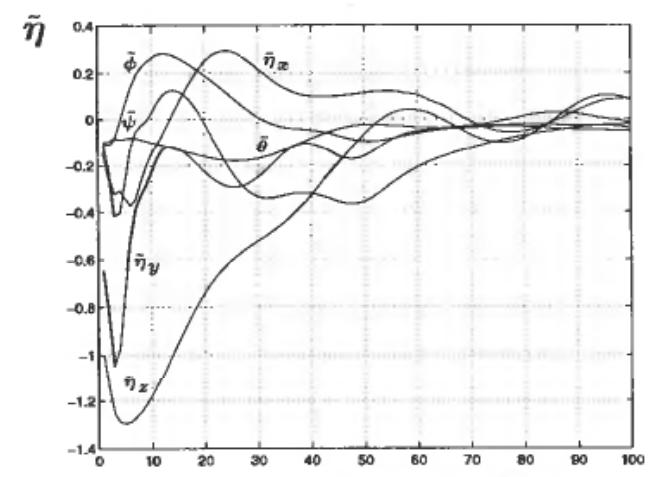

Figure 5. Simulation of the vehicle position/attitude error using (20).

$$
J_{5}=\sum_{i=1}^{k}\left|\tau_{3_{i}}\right|
$$

The performance and energy consumption indices using the controllers (18) and (20) are shown in Table 2.

Comment 1. The performance indices in Table 2 were almost equal for the two controllers but the total energy consumption was larger using the second controller (20). The reason for this is that the control vector has to counteract the hydrodynamical forces to be able to change the system momentum, in contrast to control of spacecraft-system where the control vector directly changes the system momentum.

The robustness to model uncertainties was evaluated by including uncertainties in the manipulator dynamics and in the vehicle dynamics. Two cases of uncertainty were considered;

- Case 1. A $20 \%$ uncertainty was added to the hydrodynamical coefficients. The vehicle damping was assumed to be linear and the manipulator damping was assumed to be linear and quadratic, excluding the vortex shedding effect. The indices are shown in Table 3.

- Case 2. The vehicle and manipulator damping matrix was assumed to be constant. The added mass and added moment of inertia coefficients were assumed to be completely unknown and the reaction forces were neglected. The indices are shown in Table 4. 


\begin{tabular}{rrrrrr}
\hline & $J_{1}$ & $J_{2}$ & $J_{3}$ & $J_{4}$ & $J_{5}$ \\
\hline$a_{y_{1}}$ & 0.44 & 0.41 & 156 & 6 & 185 \\
$a_{y_{2}}$ & 0.44 & $0 \cdot 30$ & 190 & 76 & 176 \\
\hline
\end{tabular}

Table 2. Performance and energy consumption indices assuming completely known model.

\begin{tabular}{cccccc}
\hline & \multicolumn{5}{c}{ Case 1 } \\
\cline { 2 - 6 } & \multicolumn{1}{c}{$J_{1}$} & $J_{2}$ & $J_{3}$ & $J_{4}$ & $J_{5}$ \\
\hline$a_{y_{1}}$ & 0.56 & 0.49 & 189 & 23 & 231 \\
$a_{y_{2}}$ & 0.7 & 0.46 & 209 & 91 & 226 \\
\hline
\end{tabular}

Table 3. Performance and energy consumption indices assuming $20 \%$ uncertainty in the model.

\begin{tabular}{cccccc}
\hline & \multicolumn{5}{c}{ Case 2 } \\
\cline { 2 - 6 } & $J_{1}$ & $J_{2}$ & $J_{3}$ & $J_{4}$ & $J_{5}$ \\
\hline$a_{y_{1}}$ & 2.6 & 1.2 & 188.9 & 180 & 297.7 \\
$a_{y_{2}}$ & 1.45 & 0.9 & 158.6 & 93 & 329 \\
\hline
\end{tabular}

Table 4. Performance and energy consumption indices assuming unknown model.

\begin{tabular}{cccccl}
\hline & $J_{1}$ & $J_{2}$ & $J_{3}$ & $J_{4}$ & \multicolumn{1}{c}{$J_{5}$} \\
\hline$a_{y_{1}}$ & 0.48 & 0.43 & 168 & $5 \cdot 3$ & 175 \\
$a_{y_{2}}$ & 0.52 & 0.33 & 202 & 78 & $167 \cdot 6$ \\
\hline
\end{tabular}

Table 5. Performance and energy consumption indices with drift in the vehicle motion.

Comment 2. The controller (18) was more robust to small uncertainty in the dynamic model while the controller (20) was more robust to large uncertainty in the dynamic model. The reason for this is that the control law (20) includes the dynamic term $\boldsymbol{P}_{01}$ while the decoupling of the end-effector velocity and vehicle velocity is purely a kinematic decoupling.

Robustness of the manipulator controller to unpredicted vehicle motion was investigated by adding a constant signal to the vehicle position/attitude vector.

Comment 3. Unpredicted drift in the vehicle motion resulted in almost the same performance for the two controllers. The energy consumption was lower using the first controller (18) since the end-effector was controlled in the I-frame.

\section{Conclusions}

In this paper two decoupling schemes suggested in (Egeland and Sagli, 1993) for control of spacecraft systems have been applied to an underwater vehicle-manipulator system. The closed-loop systems have been studied and performance, energy 
consumption and robustness have been evaluated through a simulation study. The simulation study has shown that the performance indices are almost the same for the two control schemes. With high accuracy in the dynamic model the energy consumption is lower with the conventional scheme, but for systems with large model uncertainty the scheme of decoupling the end-effector velocity from the system momentum (20) is more robust. The conventional scheme is shown to be more robust to drift in the vehicle motion.

\section{REFERENCES}

DuBowsky, S. and Papadopoulos, E. (1993). The kinematics, dynamics, and control of free-flying, and free-floating space robotic systems. IEEE Transactions on Robotics and Automation, 9 (5), 531-543.

EGELAND, O. and SAGLI, J. R. (1993). Coordination of motion in a spacecraft/manipulator system. Int. Journal of Robotics Research. Vol. 12, No. 4, Aug. 1993, pp. 366-379.

Fossen, T. I. (1991). Nonlinear Modelling and Control of Underwater Vehicles. PhD thesis. Norwegian Institute of Technology, University of Trondheim, Trondheim, Norway.

Fossen, T. I. (1994). Guidance and Control of Ocean Vehicles (John Wiley \& Sons Ltd).

HugheS, P. (1986). Spacecraft Attitude Dynamics. (John Wiley \& Sons Ltd).

SAGLI,J.R. (1991). Coordination of Motion in Manipulators with Redundant degrees of freedom. $\mathrm{PhD}$ thesis. The Norwegian Institute of Technology, University of Trondheim, Norway.

SCHJølBERG, I. and FosSEN, T. I. (1994). Modelling and control of underwater vehiclemanipulator systems. In: Proc. of the 3rd Int. Conf. on Manoeuvring and Control of Marine Craft. Southampton, England.

Spong, M. W. and VidYASAGAR, M. (1989). Robot Dynamics and Control (John Wiley \& Sons Ltd). 